\title{
The relationship between sustained attention and aerobic fitness in a group of young adults
}

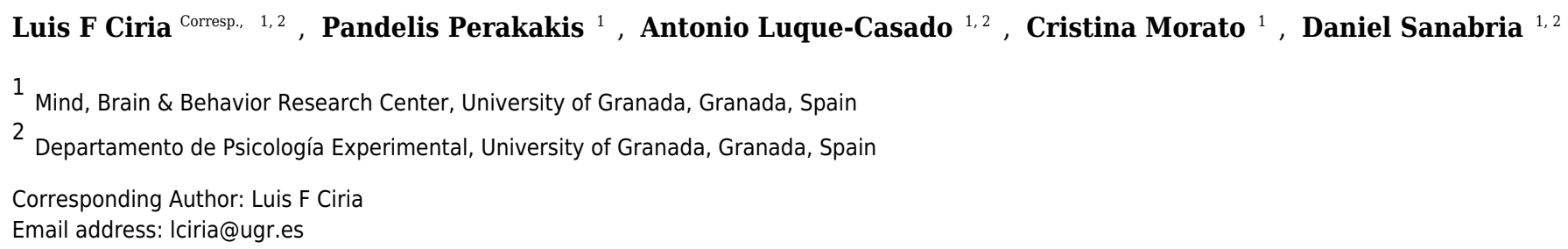

Background. A growing set of studies has shown a positive relationship between aerobic fitness and a broad array of cognitive functions. However, few studies have focused on sustained attention, which has been considered a fundamental cognitive process that underlies most everyday activities. The purpose of this study was to investigate the role of aerobic fitness as a key factor in sustained attention capacities in young adults. Methods. Forty-four young adults (18-23 years) were divided into two groups as a function of the level of aerobic fitness (high-fit and low-fit). Participants completed the Psychomotor Vigilance Task (PVT) and an oddball task where they had to detect infrequent targets presented among frequent non-targets. Results. The analysis of variance (ANOVA) showed faster responses for the high-fit group than for the low-fit group in the PVT, replicating previous accounts. In the oddball task, the high-fit group maintained their accuracy (ACC) rate of target detection over time, while the low-fit group suffered a significant decline of response ACC throughout the task. Discussion. Importantly, the results show that the greater sustained attention capacity of high-fit young adults is not specific to a reaction time (RT) sustained attention task like the PVT, but it is also evident in an ACC oddball task. In sum, the present findings point to the important role of aerobic fitness on sustained attention capacities in young adults. 


\section{The relationship between sustained attention and aerobic fitness in a}

\section{2 group of young adults.}

3 Luis F. Ciria ${ }^{1,2 *}$, Pandelis Perakakis ${ }^{1}$, Antonio Luque-Casado ${ }^{1,2}$, Cristina Morato ${ }^{1}$, Daniel

$4 \quad$ Sanabria $^{1,2}$,

5

61 Mind, Brain \& Behavior Research Center, Universidad de Granada, Granada, Spain

72 Departamento de Psicología Experimental, Universidad de Granada, Granada, Spain

9 Corresponding author:

10 Luis F. Ciria ${ }^{1}$

11 C/ Campus Universitario la Cartuja, Granada, 18071, Spain.

12 E-mail address: 1ciria@ugr.es 


\section{Abstract}

Background. A growing set of studies has shown a positive relationship between aerobic

22 fitness and a broad array of cognitive functions. However, few studies have focused on sustained

23 attention, which has been considered a fundamental cognitive process that underlies most

24 everyday activities. The purpose of this study was to investigate the role of aerobic fitness as a

25 key factor in sustained attention capacities in young adults.

26 Methods. Forty-four young adults (18-23 years) were divided into two groups as a

27 function of the level of aerobic fitness (high-fit and low-fit). Participants completed the

28 Psychomotor Vigilance Task (PVT) and an oddball task where they had to detect infrequent

29 targets presented among frequent non-targets.

30 Results. The analysis of variance (ANOVA) showed faster responses for the high-fit

31 group than for the low-fit group in the PVT, replicating previous accounts. In the oddball task,

32 the high-fit group maintained their accuracy (ACC) rate of target detection over time, while the

33 low-fit group suffered a significant decline of response ACC throughout the task.

Discussion. Importantly, the results show that the greater sustained attention capacity of

35 high-fit young adults is not specific to a reaction time (RT) sustained attention task like the PVT,

36 but it is also evident in an ACC oddball task. In sum, the present findings point to the important

37 role of aerobic fitness on sustained attention capacities in young adults. 


\section{Introduction}

Sustained attention is a fundamental high-order function that enables to direct and focus cognitive activity on specific stimuli over prolonged periods of time, and is required in order to complete any planned activity, sequenced action, or thought. Sustained attention is therefore the basis for many types of information processing and it is crucial for healthy cognitive

47 development that allows both, proper adaptation to environmental demands, and the capacity to modify behavior (Sarter, Givens \& Bruno, 2001). Importantly, sustaining attention for long periods of time is necessary in fundamental everyday activities, such as attending to academic lessons at school or driving, and in highly responsible professional tasks like performing surgery or piloting (Di Stasi et al., 2015; Suess, Porges \& Plude, 1994).

It is well known that the capacity to stay focused on a particular task is limited, which results in performance decrements with increasing time-on-task. This effect is known as the vigilance decrement and is thought to reflect the depletion of attentional resources over time (Helton \& Warm, 2008; Davies \& Parasuraman, 1982). Therefore, a profound understanding of the factors influencing sustained attention capacities is highly relevant to both theoretical and applied research.

In the present study, we focus on aerobic fitness as a key feature in young adults related to their ability to sustain attention. Although a substantial amount of studies have investigated the effects of aerobic fitness on cognitive performance in general (Colcombe \& Kramer, 2003; Hillman, Erickson \& Kramer, 2008), the relation between fitness and sustained attention has not received proper attention by exercise and cognition researchers. Here, we addressed this issue by comparing behavioral performance of high-fit and low-fit young adults in two sustained attention 64 tasks. 
65

66

67

68

69

In general, the limited previous evidence has indicated that aerobic fitness and sustained attention are positively related across the life span (Bunce, 2001; Prakash et al., 2011). For instance, in a recent study, Getzmann and collaborators (2013) found a higher susceptibility to distraction over time for inactive seniors than their active counterparts. Similar outcomes have been seen in children (Chaddock et al., 2012; Pontifex et al., 2012). With respect to young adults, to the best of our knowledge, there are only three published studies (Luque-Casado et al., 2013; Luque-Casado et al., 2016a; Luque-Casado et al., 2016b) that have reported significant differences in sustained attention capacities as a function of aerobic fitness (see Bunce, Barrowclough \& Morris, 1996, for a null result). Luque-Casado and collaborators (2013) reported the first direct evidence of a selective association between aerobic fitness and sustained attention in young adults. They found overall faster responses in a short (i.e.10 minutes) Psychomotor vigilance task (PVT) in high fit as compared to lower-fit participants while no differences were observed in a temporal orienting task and a duration perception task. Recently, the same research group (Luque-Casado et al., 2016a; Luque-Casado et al., 2016b) has extended their findings identifying potential neural and autonomic physiological mechanisms underlying the fitness-related improvements in vigilance performance in a 60' version of the PVT. Their findings showed that a higher level of fitness was related to a better behavioural performance (i.e., in the first half of the task), that was accompanied by larger amplitude in the contingent negative variation $(\mathrm{CNV})$ potential and enhanced cardiac orienting reflex. Crucially, high-fit individuals also maintained larger P3 amplitude throughout the task compared to low-fit, who showed a reduction in the P3 magnitude over time. The authors finally concluded that a higher level of fitness level was related to electrophysiological activity suggestive of better ability to allocate attentional resources over time and a greater attentive preparatory state. 

and reliable sustained attention reaction time (RT) task that requires participants to react as fast as possible to the onset of a visual (or auditory) stimulus presented on every trial and that occurs

91 at random inter-stimulus intervals. The task is characterized by the high temporal uncertainty of

92 stimuli and the low learning effects (Dinges et al., 1997; Jewett \& Kronauer, 1999). The PVT has

93 been commonly used to study the vigilance decrement by the repeated administration of the task

94 in different moments of the experimental session (Arnal et al., 2015; Van Dongen et al., 2003).

Crucially, together with RT tasks like the PVT, sustained attention has been measured by means of accuracy (ACC) tasks, typically involving the presentation of infrequent and unexpected targets presented among frequent non-target stimuli for relatively long periods of time (Bunce, 2001; Getzmann, Falkenstein \& Gajewski, 2013; Yagi et al., 1999; Pontifex et al., 2015). In fact, the first approximation to sustained attention study using this type of task was the Mackworth Clock test (Mackworth, 1948). In this task, participants watched a second by second clock that occasionally click forward two seconds instead of one. Participants were asked to

102 press a button whenever they notice the rare "two-seconds" event. Since this first study, ACC

103 oddball tasks have been widely used in cognitive science to study sustained attention

104 (Parasuraman \& Mouloua, 1987; García-Larrea, Lukaszewicz \& Mauguiére, 1992). These unspeeded tasks reduce motor demands and increase the uncertainty of the target onset. In fact, together with temporal uncertainty, the low probability of target appearance has been shown to

107 be one of the major factors to tax sustained attention (Parasuraman \& Mouloua, 1987). It is

108 important to note that this is in line with the traditional view of sustained attention as a highorder cognitive function that enables the accurate detection of infrequent and unexpected targets

110 presented in a sequence of frequent non-targets (Norman \& Shallice, 1986; Stuss et al., 1995). 
111 Luque-Casado and collaborators $(2013 ; 2016 \mathrm{a} ; 2016 \mathrm{~b})$ findings were based on the results

112 from a RT task (i.e. PVT) where participants have to respond to every stimulus. Thus, an

113 important issue remains unsolved: the potential association between aerobic fitness and the

114 ability to detect and respond to relevant non-frequent target stimuli ignoring frequent irrelevant

115 non-target stimuli, which, as noted above, has been considered one of the critical features to tax

116 sustained attention. That is, there is still the question of whether the superior performance of

117 high-fit individuals with respect to low-fit participants observed in an RT sustained attention task

118 like the PVT would be also revealed in an ACC oddball task.

119 To address the aforementioned shortcoming of previous research, here, we compared

120 performance of two groups of young adults differentiated in terms of aerobic fitness (high-fit and

121 low-fit) in the RT PVT and an ACC oddball task. The 27' duration of the oddball task allowed us

122 to investigate the vigilance decrement as a function of aerobic fitness. It is important to note that

123 previous studies have found significant vigilance decrements with even shorter task durations.

124 For example, Nuechterlein and collaborators (1983) examined sustained attention performance in

125 an oddball paradigm lasting only 8 minutes, and found a marked decrement in sensitivity over

126 time. More importantly, Ariga and Lleras (2011) found a significant vigilance decrement in

127 young adults after the first 10' of the same oddball task used in the present study. A 5' PVT

128 presented before and after the oddball task was used to investigate the changes in RT

129 performance as a function of time and aerobic fitness, and to try and replicate Luque-Casado et

130 al.'s (2013; 2016a) previous results. A shorter (with respect to Luque-Casado et al., 2013)

131 version of the PVT was used in order not overload to the experimental session, as the main focus

132 of the study was the oddball task. Note also that previous research has tested the reliability of this

133 shorter version of the PVT (Loh et al., 2004; Roach et al., 2006). 
135 low-fit participants. Similarly, we hypothesized that low-fit young adults would exhibit a larger

136 accuracy and RT impairment along the time in both tasks than their high-fit counterparts.

\section{Materials \& Method}

138 Participants

A minimum sample size of 22 participants per group was required for a power level of

140.80 as determined by an a priori power analysis based on data from a previous study (Luque-

141 Casado et al., 2013). Thus, forty-four volunteer undergraduate students were recruited through

142 announcements on billboards of the University campus at the University of Granada, Spain, to be

143 part of this study. Twenty-two participants (11 females $)^{1}$ with a low level of aerobic fitness were

144 allocated to the low-fit group and twenty-two (11 females) participants with a high level of

145 aerobic fitness were allocated to the high-fit group. The participants in the high and low-fit group

146 met the inclusion criteria of reporting at least 8 hours of training per week or less than 2 hours,

147 respectively. The ventilator aerobic threshold (VAT) of the subjects was then determined by an

148 incremental effort test in order to confirm their aerobic fitness characteristics (see below). All

149 participants had normal or corrected to normal vision, reported no neurological, cardiovascular

150 or musculoskeletal disorders and were taking no medication. All subjects gave written informed

151 consent before the study and received 10 euros for their participation. This study was approved

152 by the Ethics Committee on Human Research of the University of Granada, Spain (No. 689) and

153 complies with the ethical standards laid down in the 1964 Declaration of Helsinki.

\footnotetext{
${ }^{1}$ Note that a previous study showed that performance in the PVT did not depend on gender (Ballester et al., 2015). We therefore decided to include the same number of males and females only for the purpose of experimental control.
} 
155 Apparatus and materials

All participants were fitted with a Polar RS800 CX monitor (Polar Electro Öy, Kempele,

157 Finland) to record their heart rate (HR) during the incremental exercise test. We used a ViaSprint

$158150 \mathrm{P}$ cycle ergometer (Ergoline $\mathrm{GmbH}$, Germany) to induce physical effort and to obtain power

159 values, and a JAEGER Master Screen gas analyser (CareFusion GmbH, Germany) to provide a

160 measure of gas exchange during the effort test. All tasks were completed using a 15'6-inch LCD

161 HP laptop PC and the E-Prime software (Psychology Software Tools, Pittsburgh, PA, USA) was

162 used for stimulus presentation and data collection. The center of the laptop screen was situated at

$16360 \mathrm{~cm}$, using a chin cup, from the participants' head. The device used to collect responses was

164 the PC keyboard.

\section{Procedure}

166 The study procedure consisted of a single testing session of 90 min approximately. At the

167 beginning, we performed an anthropometric evaluation of each participant (height, weight and

168 body mass index [BMI]) in a small, dimly lit room. Then, participants completed, in this order,

169 the PVT (5 minutes), a visual oddball task (27 minutes) and the PVT (5 minutes). All cognitive

170 tasks were completed at rest with a break of 5 minutes before and after the oddball task. The

171 tasks are detailed in the following sections.

172 VAT determination test

173 At the end of the session, all subjects performed an incremental cycle-ergometer test in

174 order to determine their aerobic fitness. The incremental effort test started with a 3 minutes

175 warm-up at 30 Watts (W), with the power output increasing $10 \mathrm{~W}$ every minute. Each participant

176 set his preferred cadence (between $60-90 \mathrm{rev} \cdot \mathrm{min}^{-1}$ ) during the warm-up period and was asked

177 to maintain this cadence during the entire protocol. The test began at $60 \mathrm{~W}$ and was followed by 
178 an incremental protocol of $30 \mathrm{~W}$ every 3 minutes. Each step of the incremental protocol

179 consisted of 2 minutes of stabilized load and 1 minute of progressive load increase (5 W every

18010 seconds). The oxygen uptake $\left(\mathrm{VO} 2 \mathrm{ml} \bullet \mathrm{min}^{-1} \bullet \mathrm{kg}^{-1}\right)$, respiratory exchange ratio (RER; i.e.,

$181 \mathrm{CO}_{2}$ production $\bullet \mathrm{O}_{2}$ consumption $\left.{ }^{-1}\right)$, relative power output $\left(\mathrm{W} \cdot \mathrm{Kg}^{-1}\right)$ and heart rate $(\mathrm{bpm})$ were

182 continuously recorded throughout the test.

183 We used the ventilatory anaerobic threshold (VAT) as a reference to determine the fitness

184 level of the participants. VAT is considered to be a sensitive measure for evaluating aerobic

185 fitness and cardiorespiratory endurance performance (Londeree, 1997; Wasserman, 1984). It was

186 defined as the VO2 at the time when RER exceeded the cut-off value of 1.0 (Davis et al., 1976;

187 Yeh et al., 1983) and did not drop below that level during the 2 minutes constant load period or

188 during the next load step, never reaching the 1.1 RER. The submaximal cardiorespiratory fitness

189 test ended once the VAT was reached.

190 Psychomotor Vigilance Task

191 A modified version of the PVT developed by Wilkinson \& Houghton (1982) was used

192 here. A black circle with a red edge $\left(6.68^{\circ} \times 7.82^{\circ}\right)$ was displayed at the center of the screen in a

193 black background. The circumference began to be filled in a red color and in a counter-clockwise

194 direction with an angular velocity of 0.094 degrees per second in a random time interval (from

1952000 to $10000 \mathrm{~ms}$ ) after the appearance of the black circle. After that, the participants had a total

196 time limit of $1500 \mathrm{~ms}$ to respond. Participants were instructed to press the space bar with the

197 index finger of their dominant hand as fast as possible when the circumference started to be

198 filled, avoiding anticipation. Feedback of the response time was displayed on the screen on each

199 trial for $300 \mathrm{~ms}$. Data from trials with RTs below $100 \mathrm{~ms}(2.72 \%)$ were considered anticipations

200 and therefore discarded from the analysis. Following the response to the target, or after $1500 \mathrm{~ms}$ 
201 in case of a missed response (if a response was not made during this time, the message "You did 202 not answer" appeared on the screen), the next trial began. The task lasted for 5 minutes without 203 interruptions.

204 Oddball task

A visual oddball task based on that used by Ariga \& Lleras (2011) was designed to measure sustained attention. The task consisted of a sequence of vertical, white and one-pixel 207 thick lines displayed in the center of screen on a black background. A red circle was continuously visible at the center of the display as fixation point. The participants were instructed to respond to the target line (short line, $10.47^{\circ}$ ) pressing the "B" key with their index finger of their dominant hand and not to respond when the non-target line (long line, $11.99^{\circ}$ ) was

211 shown. Participants were encouraged to respond accurately, not quickly. The task consisted of

212 two blocks of 13.5 minutes, without any break between them, with the target stimuli pseudo-

213 randomly presented in $10 \%$ of trials (with the restriction that thirty targets appeared on each

214 block) and non-target stimuli in $90 \%$. The stimulus duration was $153 \mathrm{~ms}$, and a variable

215 interstimulus interval (1.3-2.3 s) was used. The task lasted for 27 minutes without breaks.

\section{Design Statistical analyses}

218 samples.

For the PVT, a two-way analysis of variance (ANOVA) with the between-participants factor of aerobic fitness (high-fit, low-fit) and the within-participants factors of session (pre,

221 post) was applied to analyze participants' mean RT. 
223 between- subject factor and time-on-task (block 1, block 2) as within-subject factor was used to

224 analyze (separately) participants' ACC (hits) and RTs to target trials, and ACC (commission

225 errors) to non-target trials.

All analyses were performed with the SPSS statistical package. Multiple contrasts using $\mathrm{t}$

$230\left(\eta p^{2}\right)$ for Fs and as Cohen's d for t-tests.

\section{Results}

Descriptive and fitness data

the incremental test parameters (i.e., maximum power output $(\mathrm{W}), t(42)=5.437, p<.001, d=$ 1.67, relative power output $\left(\mathrm{W} \bullet \mathrm{kg}^{-1}\right), t(42)=5.488, p<.001, d=1.69, \mathrm{VO}_{2}\left(\mathrm{~mL} \cdot \mathrm{min}^{-1} \bullet \mathrm{kg}^{-1}\right), t$ $(42)=4.595, p<.001, d=1.41$, and HR $(\mathrm{bpm}), t(42)=-3.987, p<.001), d=-1.25$. Data showed clear evidence of the difference in aerobic fitness between groups (see Table 1). There were no statistically significant differences between groups in any other descriptive data (all $p s \geq$ $239 \quad .5)$

Table 1. Mean and standard deviation of descriptive and fitness data for the high-fit and low-fit groups. 


\section{Anthropometrical characteristics}

Sample size (n)

22 (11 females)

22 (11 females)

Age (years)

Height $(\mathrm{cm})$

Weight $(\mathrm{kg})$

BMI $\left(\mathrm{kg} / \mathrm{m}^{2}\right)$

Incremental test parameters

Power max at VAT (W)

Relative power at VAT (W/kg)

$\mathrm{VO}_{2}$ at $\mathrm{VAT}(\mathrm{ml} / \mathrm{kg} / \mathrm{min})$

Heart rate (bpm)
$22.3 \pm 3.5$

$170 \pm 7.8$

$66.2 \pm 9.9$

$22.7 \pm 2,7$

$195 \pm 70.4$

$2.9 \pm 1.1$

$35.9 \pm 13.9$

$61.7 \pm 12.7$
$22.5 \pm 3.3$

$168 \pm 10.8$

$67.2 \pm 16.8$

$23.3 \pm 3.9$

PVT

242 The ANOVA of participants' mean RTs revealed a marginally significant main effect of aerobic

243 fitness, $F(1,42)=3.663, p=.052, \eta p^{2}=.08$. The high-fit group responded faster overall than the

244 low-fit group (323 $\pm 24 \mathrm{~ms}$ and $346 \pm 46 \mathrm{~ms}$, respectively). Neither the main effect of session,

$245 F<1$, nor the interaction between both factors reached statistical significance, $F<1$.

\section{Oddball task}

247 Hits trials

248 The analysis of ACC (Hits) for target-trials yielded a significant main effect of time-on-task, $F$

$249(1,42)=14.207, p<.001, \eta p^{2}=.25$, that was better qualified by the significant interaction

250 between aerobic fitness (high-fit, low-fit) and time-on-task (block 1, block 2), $F(1,42)=4.502, p$ 
$251=.040, \eta p^{2}=.09$; see Figure 1). The t-test for dependent samples between block 1 and block 2

252 reached significance for the low-fit group, $t(21)=4.069, p<.001, d=0.48$, showing a reduced

253 ACC over time (81.2 $\pm 17 \%$ and $72.4 \pm 18 \%$, in block 1 and block 2 , respectively). In contrast,

254 the difference between blocks was not significant for the high-fit group, $t(21)=1.194, p=.246$, $255 d=0.17),(82.3 \pm 12 \%$ and $80.1 \pm 15 \%$, in block 1 and block 2 , respectively). The analysis of 256 RTs for target-trials yielded no significant main effects of time-on-task, $F<1$, and aerobic 257 fitness, $F(1,42)=2.855, p=.099, \eta p^{2}=.06$. The interaction between both factors was not 258 significant either, $F<1$.

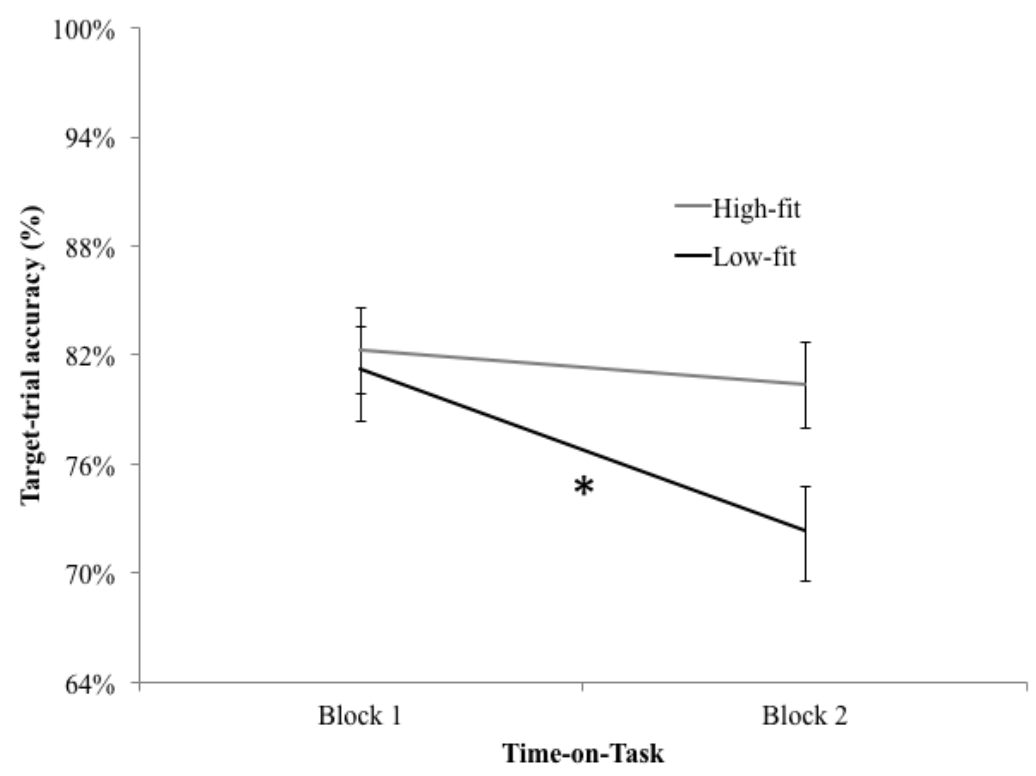

259

Figure 1. Mean response ACC (\%) and standard error for target-trials as a function of

261 Group and Block. * Significant effect of time-on-task

Commission errors

264 The maintenance of accuracy in target-trials during the task showed by high-fit participants

265 could have been due to a mere higher response rate than low-fit participants. However, this 
266 would have resulted also in more commission errors (responses to non-targets). To address this

267 issue, we analyzed participants' mean ACC (commission errors) to non-targets. The ANOVA

268 showed a main effect of time-on-task, $F(1,42)=7.579, p=.009, \eta p^{2}=.15$, with participants

269 being more accurate in block $2(96.3 \pm 12 \%$ and $92.3 \pm 11 \%$, for high-fit and low-fit,

270 respectively) than in block $1(95.1 \pm 12 \%$ and $88.8 \pm 15 \%$, for high-fit and low-fit,

271 respectively). The main effect of aerobic fitness failed to reach statistical significance, $F(1,42)=$

$2723.680, p=.062, \eta p^{2}=.08$. There was no significant interaction between both factors $F(1,42)=$

$2731.549, p=.220, \eta p^{2}=.03$.

\section{Discussion}

In the present study, we investigated the role of aerobic fitness in the sustained attention capacity of young adults. Towards this aim, two groups of participants (high-fit and low-fit) completed the PVT and an oddball task.

The results of the PVT showed that high-fit young adults were faster than low-fit, replicating previous research (Luque-Casado et al., 2013; Luque-Casado et al., 2016a; Luque-

280 Casado et al., 2016b). Interestingly, even though the statistical analysis showed a marginal ( $p=$

281.053 ) effect, the $23 \mathrm{~ms}$ difference between high-fit and low-fit participants was larger than the 19

282 ms reported in Luque-Casado et al. (2013) and the $12 \mathrm{~ms}$ (in the first 10' of the task) reported in

283 Luque-Casado et al. (2016a). Taken together, the present and previous research from our

284 laboratory supports the PVT as a suitable tool to measure group differences in terms of RT

285 performance in a vigilance task involving high temporal uncertainty of target onset. Note also,

286 that previous studies (e.g., Dorrian et al., 2005; Drummond et al., 2005; Lim \& Dinges, 2008)

287 showed the recruitment of the sustained attention brain network during the PVT, highlighting the sensitivity of the task to vigilance demands, over and above the time-on-task effect. Indeed, 
289 Drummond et al., (2005) found different activation patterns of cortical and subcortical brain 290 regions during the fastest RTs of a PVT, relative to the slowest RTs. They suggested that the 291 activity of these regions might reflect top-down modulation processes related to the capacity to

292 maintain the focus on the task. In the present study, high-fit and low-fit participants appear to 293 differ in their overall RT performance, but not in the effect of session. The lack of a session

294 effect in the PVT might be due to the time break before the second PVT and even because of the 295 presence of the oddball task between PVT sessions, which might have provoked a sort of "mind 296 reset" (cf. Ariga \& Lleras, 2011), preventing vigilance degradation with respect to the first PVT.

297 Nevertheless, it is also possible that the PVT employed in the current manuscript was not 298 demanding sustained attention enough to induce the RT increment from the first to the second 299 PVT session.

300 Importantly, in the oddball task, the high-fit group maintained their ACC rate over the 301 whole duration of the task, while the low-fit group suffered a significant decline of response

302 ACC over time. This finding suggests that aerobic fitness is related to the capacity to focus on 303 relevant infrequent stimuli ignoring irrelevant frequent stimuli. Interestingly, the lack of group

304 differences in RT in the oddball task seems to indicate that the greater sustained attention 305 capacity showed by high-fit participants was irrespective of the nature of the task, i.e., high-fit 306 young adults seem to adapt their performance to the goals of each task, either speed or ACC.

307 Note, though, that the task instruction (stressing ACC over RT) in the oddball task might also 308 explain the absence of RT difference between groups.

309 The present findings could be interpreted in the context of the "cardiovascular

310 hypothesis" which proposes that exercise, particularly aerobic exercise, results in measurable

311 gains in cardiorespiratory fitness, leads to benefits at the cognitive and brain levels. These gains 
312 refer to an increased ability of the heart to deliver oxygen to the working muscles which is

313 thought to be associated with changes in cerebral structure (Colcombe \& Kramer, 2003), cerebral

314 blood flow (Endres et al., 2003), and a brain-derived neurotrophic factor [BDNF, IGF-1]

315 (Vaynman, Ying \& Gomez-Pinilla, 2003). In turn, all these changes have themselves been shown

316 to be associated with cognitive performance (Vaynman, Ying \& Gomez-Pinilla, 2004; Hillman,

317 Erickson \& Kramer, 2008; Voss et al., 2013; McMorris, 2016). Thus, according to the

318 cardiovascular fitness hypothesis, changes in aerobic fitness are necessary for the cognitive

319 benefits of physical activity to be observed. Certainly, the higher levels of aerobic fitness of

320 high-fit young adults in our study may be associated with changes in underlying physiological

321 mechanisms that result in a greater capacity to maintain attention over time compared to low-fit

322 young adults. However, it is important to note that several meta-analyses of available studies

323 seriously question this hypothesis (Angevaren et al., 2008; Verburgh et al., 2014; Young et al.,

324 2015). This means that the exact mechanisms that produce the observed enhanced cognitive

325 performance in physically fit individuals are still unknown.

326 At this point, it is also important to note that the purpose of this study was not to establish

327 a causal-effect relationship, but rather to investigate the potential association between sustained

328 attention and aerobic fitness. Randomized controlled trials are needed to establish that cause-

329 effect relationship with respect to sustained attention and aerobic fitness. Moreover, we believe

330 that future studies should investigate complex organ interactions (e.g. brain-heart

331 communication) as a mechanism contributing to the enhanced cognitive and brain function in

332 physically fit individuals (see Perakakis et al., 2017, for a first approach to this issue).

\section{Conclusions}


between aerobic fitness and sustained attention capacity in young adults. Importantly, a profound

knowledge of the factors that support and enhance sustained attention is especially critical for

public health, as a sedentary lifestyle has been blamed for so many diseases and also

deterioration in cognitive function and performance of many daily life activities.

\section{References}

Angevaren M., Aufdemkampe G., Verhaar HJ., Aleman A., Vanhees L. 2008. Physical activity and enhanced fitness to improve cognitive function in older people without known cognitive impairment. Cochrane database syst rev 3.

Ariga A., Lleras A. 2011. Brief and rare mental "breaks" keep you focused: Deactivation and reactivation of task goals preempt vigilance decrements. Cognition 118:439-443. DOI: 10.1016/j.cognition.2010.12.007.

Arnal PJ., Sauvet F., Leger D., van Beers P., Bayon V., Bougard C., Rabat A., Millet GY., Chennaoui M. 2015. Benefits of sleep extension on sustained attention and aleep pressure before and during total sleep deprivation and recovery. Sleep 38:1935-1943. DOI: $10.5665 /$ sleep. 5244 .

Ballester R., Huertas F., Yuste FJ., Llorens F., Sanabria D. 2015. The relationship between regular sports participation and vigilance in male and female adolescents. Plos one 10:e0123898. DOI: 10.1371/journal.pone.0123898.

Bunce D. 2001. Age differences in vigilance as a function of health-related physical fitness and task demands. Neuropsychologia 39:787-797.

Bunce DJ., Barrowclough A., Morris I. 1996. The moderating influence of physical fitness on age gradients in vigilance and serial choice responding tasks. Psychology and aging 11:671.

Chaddock L., Erickson KI., Prakash RS., Voss MW., VanPatter M., Pontifex MB., Hillman CH., Kramer AF. 2012. A functional MRI investigation of the association between childhood aerobic fitness and neurocognitive control. Biological psychology 89:260-268. DOI: 10.1016/j.biopsycho.2011.10.017.

Colcombe S., Kramer AF. 2003. Fitness effects on the cognitive function of older adults a meta-analytic study. Psychological science 14:125-130.

Davies DR., Parasuraman R. 1982. The psychology of vigilance. Academic Pr.

Davis JA., Vodak P., Wilmore JH., Vodak J., Kurtz P. 1976. Anaerobic threshold and maximal aerobic power for three modes of exercise. Journal of Applied Physiology 41:544-550.

Dinges DF., Pack F., Williams K., Gillen KA., Powell JW., Ott GE., Aptowicz C., Pack AI. 1997. Cumulative sleepiness, mood disturbance and psychomotor vigilance performance decrements during aweek of sleep restricted to 4-5 hours per night. Sleep: Journal of sleep research \& sleep medicine 20:267-277. 
372

373

374

375

376

377

378

379

380

381

382

383

384

385

386

387

388

389

390

391

392

393

394

395

396

397

398

399

400

401

402

403

404

405

406

407

408

409

410

411

412

413

414

415

416

417

Di Stasi LL., Diaz-Piedra C., Suárez J., McCamy MB., Martinez-Conde S., Roca-Dorda J., Catena A. 2015. Task complexity modulates pilot electroencephalographic activity during real flights: In-flight EEG. Psychophysiology 52:951-956. DOI: 10.1111/psyp.12419.

Dorrian, J., Rogers, N. L., \& Dinges, D. F. (2005). Psychomotor vigilance performance: Neurocognitive assay sensitive to sleep loss (Doctoral dissertation, Marcel Dekker).

Drummond, S. P., Bischoff-Grethe, A., Dinges, D. F., Ayalon, L., Mednick, S. C., \& Meloy, M. J. (2005). The neural basis of the psychomotor vigilance task. Sleep, 28(9), 10591068.

Endres, M., Gertz, K., Lindauer, U., Katchanov, J., Schultze, J., Schröck, H., Nickening, G., Kuschinsky, W., Dirnagl, U., \& Laufs, U. (2003). Mechanisms of stroke protection by physical activity. Annals of neurology, 54(5), 582-590.

García-Larrea L., Lukaszewicz A-C., Mauguiére F. 1992. Revisiting the oddball paradigm. Non-target vs neutral stimuli and the evaluation of ERP attentional effects. Neuropsychologia 30:723-741. DOI: 10.1016/0028-3932(92)90042-K.

Getzmann S., Falkenstein M., Gajewski PD. 2013. Long-term cardiovascular fitness is associated with auditory attentional control in old adults: neuro-behavioral evidence. Plos one 8:e74539. DOI: 10.1371/journal.pone.0074539.

Helton WS., Warm JS. 2008. Signal salience and the mindlessness theory of vigilance. Acta psychologica 129:18-25. DOI: 10.1016/j.actpsy.2008.04.002.

Hillman CH., Erickson KI., Kramer AF. 2008. Be smart, exercise your heart: exercise effects on brain and cognition. Nature reviews neuroscience 9:58-65.

Jewett ME., Kronauer RE. 1999. Interactive mathematical models of subjective alertness and cognitive throughput in humans. Journal of biological rhythms 14:588-597. DOI: 10.1177/074873099129000920.

Lim J., Dinges DF. 2008. Sleep deprivation and vigilant attention. Annals of the new york academy of sciences 1129:305-322. DOI: 10.1196/annals.1417.002.

Loh, S., Lamond, N., Dorrian, J., Roach, G., \& Dawson, D. (2004). The validity of psychomotor vigilance tasks of less than 10-minute duration. Behavior research methods, instruments, \& computers, 36(2), 339-346.

Londeree BR. 1997. Effect of training on lactate/ventilatory thresholds: a meta-analysis: Medicine \& science in sports \& exercise 29:837-843. DOI: 10.1097/00005768-19970600000016.

Luque-Casado A., Zabala M., Morales E., Mateo-March M., Sanabria D. 2013. Cognitive performance and heart rate variability: the influence of fitness level. Plos one 8:e56935. DOI: 10.1371/journal.pone.0056935.

Luque-Casado A., Perales JC., Cárdenas D., Sanabria D. 2016a. Heart rate variability and cognitive processing: The autonomic response to task demands. Biological psychology 113:8390. DOI: 10.1016/j.biopsycho.2015.11.013.

Luque-Casado A., Perakakis P., Ciria LF., Sanabria D. 2016b. Transient autonomic responses during sustained attention in high and low fit young adults. Scientific reports 6 . DOI: $10.1038 /$ srep27556.

Mackworth NH. 1948. The breakdown of vigilance durning prolonged visual search. Quarterly Journal of Experimental Psychology 1:6-21. DOI: 10.1080/17470214808416738.

McMorris T. 2016. Developing the catecholamines hypothesis for the acute exercisecognition interaction in humans: Lessons from animal studies. Physiology \& behavior 165:291299. DOI: 10.1016/j.physbeh.2016.08.011. 
418

419

420

421

422

423

424

425

426

427

428

429

430

431

432

433

434

435

436

437

438

439

440

441

442

443

444

445

446

447

448

449

450

451

452

453

454

455

456

457

458

459

460

461

462

Norman DA., Shallice T. 1986. Attention to Action. In: Davidson RJ, Schwartz GE, Shapiro D eds. Consciousness and Self-Regulation. Springer US, 1-18.

Nuechterlein KH., Parasuraman R., Jiang Q. 1983. Visual sustained attention: image degradation produces rapid sensitivity decrement over time. Science 220:327-329. DOI: 10.1126/science.6836276.

Parasuraman R., Mouloua M. 1987. Interaction of signal discriminability and task type in vigilance decrement. Perception \& Psychophysics 41:17-22.

Perakakis, P., Luque-Casado, A., Ciria, L. F., Ivanov, P. C., \& Sanabria, D. (2017). Neural Responses to Heartbeats of Physically Trained and Sedentary Young Adults. bioRxiv. https://doi.org/10.1101/156802.

Pontifex MB., Parks AC., Henning DA., Kamijo K. 2015. Single bouts of exercise selectively sustain attentional processes. Psychophysiology 52:618-625.

Pontifex MB., Scudder MR., Drollette ES., Hillman CH. 2012. Fit and vigilant: The relationship between poorer aerobic fitness and failures in sustained attention during preadolescence. Neuropsychology 26:407-413. DOI: 10.1037/a0028795.

Prakash RS., Voss MW., Erickson KI., Lewis JM., Chaddock L., Malkowski E., Alves H., Kim J., Szabo A., White SM., Wójcicki TR., Klamm EL., McAuley E., Kramer AF. 2011. Cardiorespiratory fitness and attentional control in the aging brain. Frontiers in human neuroscience 4. DOI: 10.3389/fnhum.2010.00229.

Roach, G. D., Dawson, D., \& Lamond, N. (2006). Can a shorter psychomotor vigilance task be used as a reasonable substitute for the ten - minute psychomotor vigilance task? Chronobiology international, 23(6), 1379-1387.

Sarter M., Givens B., Bruno JP. 2001. The cognitive neuroscience of sustained attention: where top-down meets bottom-up. Brain research reviews 35:146-160.

Stuss DT., Shallice T., Alexander MP., Picton TW. 1995. A multidisciplinary approach to anterior attentional functions. Annals of the New York academy of sciences 769:191-212. DOI: 10.1111/j.1749-6632.1995.tb38140.x.

Suess PE., Porges SW., Plude DJ. 1994. Cardiac vagal tone and sustained attention in school-age children. Psychophysiology 31:17-22.

Van Dongen HP., Maislin G., Mullington JM., Dinges DF. 2003. The cumulative cost of additional wakefulness: dose-response effects on neurobehavioral functions and sleep physiology from chronic sleep restriction and total sleep deprivation. Sleep-New York then Westchester26:117-129.

Vaynman S., Ying Z., Gomez-Pinilla F. 2003. Interplay between brain-derived neurotrophic factor and signal transduction modulators in the regulation of the effects of exercise on synaptic-plasticity. Neuroscience 122:647-657. DOI: 10.1016/j.neuroscience.2003.08.001.

Vaynman S., Ying Z., Gomez-Pinilla F. 2004. Hippocampal BDNF mediates the efficacy of exercise on synaptic plasticity and cognition. European journal of neuroscience 20:25802590. DOI: 10.1111/j.1460-9568.2004.03720.x.

Verburgh L., Konigs M., Scherder EJA., Oosterlaan J. 2014. Physical exercise and executive functions in preadolescent children, adolescents and young adults: a meta-analysis. British Journal of Sports Medicine 48:973-979. DOI: 10.1136/bjsports-2012-091441.

Voss MW., Vivar C., Kramer AF., van Praag H. 2013. Bridging animal and human models of exercise-induced brain plasticity. Trends in cognitive sciences 17:525-544. DOI: 10.1016/j.tics.2013.08.001. 
463 Wasserman K. 1984. The anaerobic threshold measurement to evaluate exercise 464 performance. American review of respiratory disease 129:S35-S40. DOI:

465 10.1164/arrd.1984.129.2P2.S35.

466 Wilkinson RT., Houghton D. 1982. Field test of arousal: A portable reaction timer with 467 data storage. human factors: The journal of the human factors and ergonomics society 24:487468 493. DOI: 10.1177/001872088202400409.

469 Wilson A., Dollman J., Lushington K., Olds T. 2010. Reliability of the 5-min 470 psychomotor vigilance task in a primary school classroom setting. Behavior research methods 471 42:754-758. DOI: 10.3758/BRM.42.3.754.

472 Yagi Y., Coburn KL., Estes KM., Arruda JE. 1999. Effects of aerobic exercise and 473 gender on visual and auditory P300, reaction time, and accuracy. European journal of applied 474 physiology and occupational physiology 80:402-408.

475

476

477

478

479

Yeh MP., Gardner RM., Adams TD., Yanowitz FG., Crapo RO. 1983. "Anaerobic threshold": problems of determination and validation. Journal of applied physiology 55:11781186.

Young, J., Angevaren, M., Rusted, J., \& Tabet, N. (2015). Aerobic exercise to improve cognitive function in older people without known cognitive impairment. The Cochrane library. 